\title{
Patient comfort from the technologist perspective: factors to consider in mammographic imaging
}

\author{
This article was published in the following Dove Press journal: \\ International Journal of Women's Health \\ 18 May 2017 \\ Number of times this article has been viewed
}

\section{Christina C Mendat \\ Dave Mislan² \\ Lisa Hession-Kunz ${ }^{2}$}

'Human Factors MD, Charlotte, NC, ${ }^{2}$ Hologic Inc., Marlborough, MA, USA

Correspondence: Dave Mislan Hologic Inc., 250 Campus Drive, Marlborough, MA 0I752, USA Tel +l 78I 9997569

Email dave.mislan@hologic.com

\begin{abstract}
A sample size of 280 certified mammography technologists were surveyed to understand what factors affect patient discomfort during breast imaging. Given mammography technologists' level of patient involvement, they are uniquely positioned to observe factors that affect patient comfort. The findings suggest that according to technologists, multiple factors, including patient ethnicity, breast density, previous biopsy and lumpectomy experience, as well as psychological factors, impact breast discomfort during mammography. Additionally, with respect to imaging protocols, technologists attributed $80 \%$ of moderate-to-extreme discomfort to "length of compression time" (27\%) and "compression force" (53\%). Technologists also attributed "pinching at chest wall" and "hard edges of breast platform" to "very high" discomfort significantly more times $(P<0.05)$ than "coolness and edges of paddle". These findings confirm some of what has been reported to date and challenge other findings. Given that recent decline in breast cancer mortality has been attributed to improvements in early detection and treatment, approaches to reduce discomfort should be considered in order to promote screening compliance. Although more research is needed, it is apparent that the patient experience of comfort and pain during mammography is an area warranting increased research and solutions.
\end{abstract}

Keywords: mammography, discomfort, pain, density, compliance, breast

\section{Past discomfort research}

It is generally accepted that survival is lower among women with more advanced stages of breast cancer at diagnosis. When detected and treated early, the 5-year relative survival for localized breast cancer is $99 \%$ and for regional disease, it is $85 \%$. If the cancer has spread to distant organs, the 5-year survival drops to $26 \%{ }^{1}$ Recent decline in breast cancer mortality has been attributed to patient education, resulting in improvements in both early detection and treatment. To this end, it is critical that candidates for screening are receiving routine mammograms to assure the earliest detection of breast cancer. Yet, the US Centers for Disease Control and Prevention (CDC) ${ }^{2}$ data show that $>30 \%$ of women aged $>40$ years in the US have not had a mammogram in the past 2 years. A review of previous research ${ }^{3,4}$ shows some connection between anxiety about pain and discomfort felt during mammography and the reluctance to comply with screening guidelines. Opportunities to increase comfort during screening should be considered to encourage participation in routine screening and to increase early detection of cancer.

A number of studies have been conducted with a focus on better understanding patient discomfort during mammography. De Groot et $\mathrm{al}^{5}$ reported that a new compression procedure based on a standard mean contact area pressure reduces patient-reported pain and discomfort during mammography without sacrificing quality images or increasing radiation dose. More specifically, with the use of a pressure-based protocol, painful or 
uncomfortable overcompression in small breasts is avoided. Rutter et $\mathrm{al}^{6}$ conducted a survey-based study of women and found that the most important predictor of pain was the previous expectation of pain and that discomfort had little effect on satisfactory or subsequent screenings.

However, in a study by Kornguth et al, ${ }^{7}$ 91\% of women reported having some degree of pain during a mammogram (in the low-to-moderate range). This finding was different from the Rutter et $\mathrm{al}^{6}$ study and is likely a function of the pain scales used. However, the Kornguth et $\mathrm{al}^{7}$ study provided predictors to pain. The two variables that were shown to consistently predict pain were: 1) pain at the last mammogram and 2) breast density, which was rated on a 4-point scale from fatty to dense. These findings differ from those of Bruyninckx et $\mathrm{al}^{8}$ who found that anxiety, breast density and compression rate were not significant in contributing to patient discomfort.

In a 2000 study by Keemers-Gels et al, ${ }^{9}$ the authors carried out a study on pain that focused on various historical, sociodemographic and situational factors that may affect pain during screening. Of the women surveyed, $72.9 \%$ reported mammograms to be mild to severely painful. The factors that predicted breast pain were: 1) sensitive breasts; 2) a family history of breast disease; 3) sensitivity of breasts during the 3 days prior to the examination and 4) insufficient attention of technologists.

A more recent survey conducted by Padoan et $\mathrm{al}^{4}$ highlighted that "fear of pain" was a factor that affects screening compliance. In a study conducted by Engelman et al, ${ }^{10}$ one of the seven primary themes affecting mammography satisfaction in addition to discomfort/pain during examination was "treatment by the technologist".

With respect to investigations of the relationship between ethnicity and mammogram discomfort, different approaches have been taken. For instance, Whelehan et $\mathrm{al}^{11}$ reported that $25 \%-46 \%$ of English women cited "pain" to be the reason they did not return for their following yearly mammogram. In 2004, Asghari and Nicholas ${ }^{12}$ conducted a study aimed at understanding the coping strategies for Iranian women as it relates to pain and discomfort. This study found that women who were more apprehensive and attempted to use more coping strategies actually experienced more pain during their screening.

\section{Technologist perspective}

Past research on patient comfort and pain during breast screenings and diagnostic imaging has been mixed in terms of the factors that impact the experience. Most of the research conducted to date is from the patients' perspective. This study looked at patient comfort from the technologist's perspective, a group whose frequent contact with patients makes them uniquely qualified. Depending on breast imaging center patient volume, a mammography technologist in the US can screen $>20$ patients in a day. This experience uniquely positions technologists to observe factors that may affect patient comfort and screening compliance.

Thus, this study had three primary objectives: 1) to understand what factors may be influencing patient comfort during breast imaging; 2) to understand if discomfort during imaging may be affecting screening compliance and 3) to identify areas of opportunities for manufacturers to consider to decrease patient discomfort in an effort to increase screening compliance.

\section{Methods}

\section{Participants}

A total of 280 Mammography Quality Standards Act (MQSA)-certified mammography technologists across the US participated in this survey, of whom $20 \%$ conduct $>20$ screens/day and $50 \%$ conduct between 10 and 20 screens/day on average.

\section{Materials and procedure}

A survey was developed to understand what factors appear to technologists to affect patient discomfort during breast imaging. Areas of investigation around discomfort included: 1) ethnicity; 2) size and type of breasts (using the American College of Radiology [ACR] Breast Imaging Reporting and Data System [BI-RADS $\left.{ }^{\circledR}\right]$ categories for breast density ${ }^{13}$ ) and past history with mammograms; 3) general imaging processes (ie, holding breath, compression) and 4) psychological factors.

\section{Ethnicity}

Technologists were asked to report their perception of the level of discomfort experienced by various demographic groups during breast imaging. Technologists reported discomfort levels for the following: 1) Asian; 2) African American; 3) Hispanic; 4) non-Hispanic White; 5) younger patients aged $<50$ years and 6) older patients aged $>70$ years. With respect to discomfort ratings, a rating scale was developed that focused on the use of the term discomfort, as it has been demonstrated in past literature ${ }^{14}$ that discomfort "is more sensitive at lower noxious stimulus levels". The following discomfort scale was used: no discomfort, little discomfort, some discomfort, moderate discomfort, somewhat high discomfort, high discomfort, very high discomfort and extreme discomfort. 


\section{Size and type of breasts and mammogram history}

Technologists provided discomfort ratings for patients with various types of breasts: 1) small-breasted patients (permits imaging with smaller compression paddle); 2) large-breasted patients (requires larger compression paddle); 3) densebreasted patients (BI-RADS rating C or D); 4) fatty-breasted patients (BI-RADS rating A or B); 5) patients with implants and 6) patients with prior biopsies or lumpectomies. The rating scale included no discomfort, little discomfort, some discomfort, moderate discomfort, somewhat high discomfort, high discomfort, very high discomfort and extreme discomfort.

\section{Imaging processes}

The standard mammogram comprises the same basic steps, regardless of breast size, type or other physical characteristics of the patient. Technologists were asked to rate the level of patient discomfort experienced as a function of this protocol (ie, using the scale very low, low, neutral, high or very high). Of particular interest were: 1) the requirement to "stop breathing"; 2) the length of time under compression; 3) compression force and 4) breast sticking to the platform.

\section{Psychological factors}

Technologists were asked to rate how much impact "proper communication of exam expectations" would have on patient discomfort (ie, using the scale very low, low, neutral, high or very high) in addition to other considerations such as mood lighting, softer paddles, calming music and aromatherapy.

\section{Data analyses}

Kruskal-Wallis tests (a nonparametric alternative to the analysis of variance [ANOVA]) were conducted on all data to test for significant differences. Mann-Whitney $U$ tests (for multiple comparisons) were used to determine direction of significance where applicable.

\section{Results and discussion Discomfort as a function of ethnicity and age}

Younger patients were reported to have significantly more discomfort (moderate-to-extreme discomfort) than the other groups $(P<0.05$; Figure 1). Figure 1 provides the number of technologists reporting specific demographic groups experiencing "moderate-to-extreme" amounts of discomfort during screening.

Among the different ethnic groups, Asian women were reported to be most sensitive. This may be attributed to, in part, the nature of most Asian women's breasts. Technologists report this group to be "dense" in nature. This would be consistent with the Kornguth et $\mathrm{al}^{7}$ finding that breast density is a primary predictor of breast discomfort during imaging.

\section{Discomfort as a function of breast type}

Consistent with findings regarding Asian women, technologists reported dense-breasted patients experienced discomfort at a significantly higher level $(P<0.05)$ than the other groups with the exception of those women who have had prior procedures (Figure 2). A similar trend was found between women with small breasts versus large breasts, whereby small-breasted women were reported to have more discomfort than large-breasted women; however, it was not statistically significant $(P=0.08)$. Figure 2 provides the number of technologists reporting specific breast types experiencing "moderate-to-extreme" amounts of discomfort during screening. Technologists reported that patients who

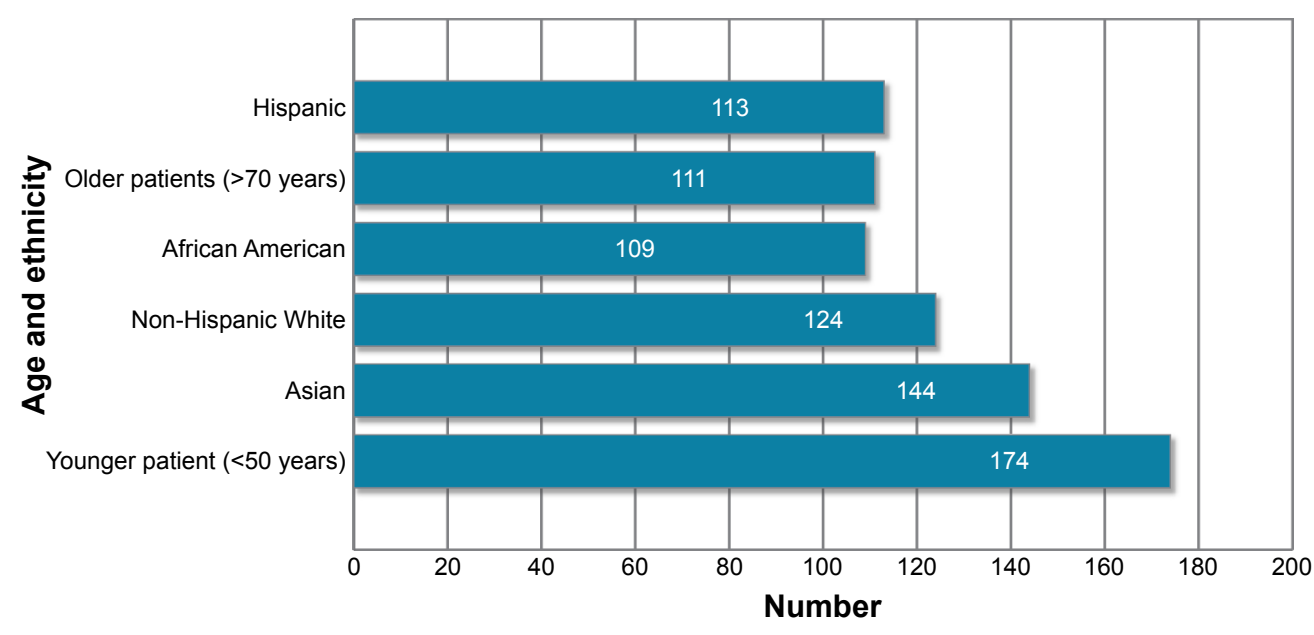

Figure I Number of technologists reporting patients in specific demographic groups to have "moderate-to-extreme" discomfort during screening. 


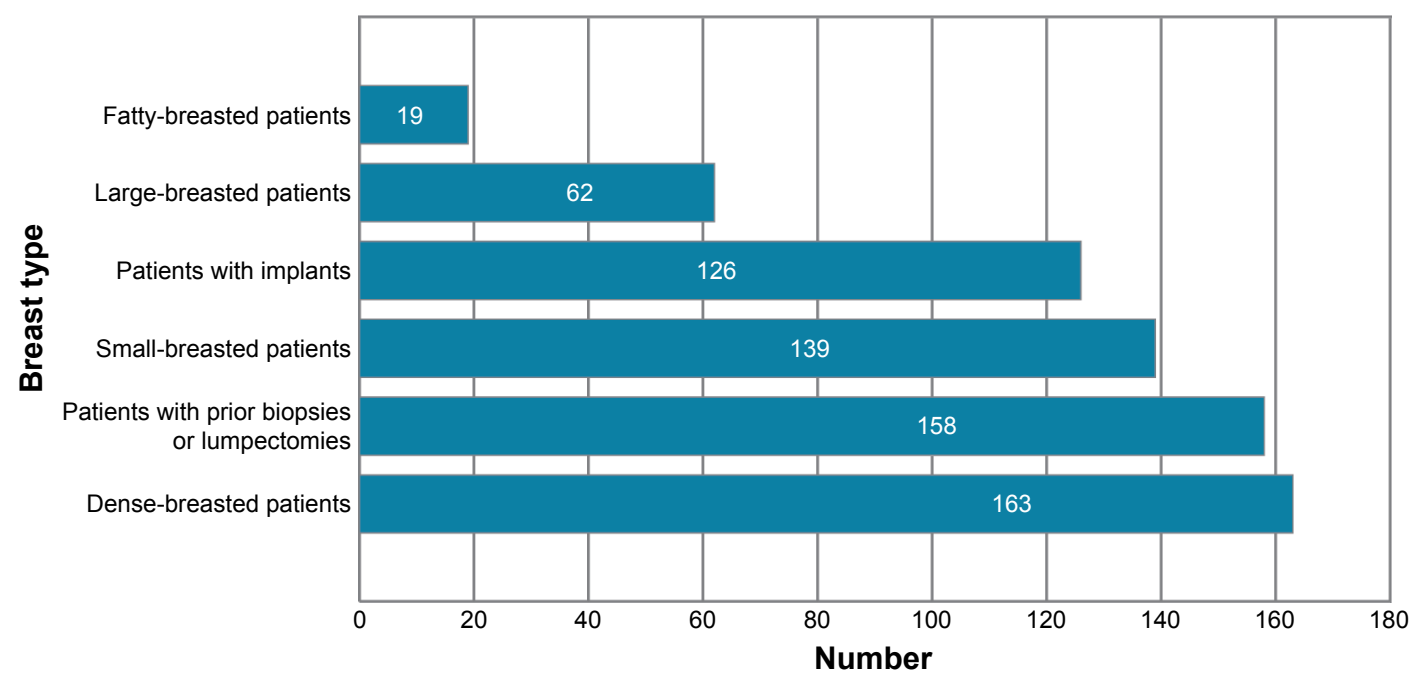

Figure 2 Number of technologists reporting patients with specific breast types to have "moderate-to-extreme" discomfort during screening.

have had prior biopsies and lumpectomies have a similar amount of discomfort to women with dense breasts.

\section{Discomfort as a function of imaging protocols}

Technologists reported that $80 \%$ of moderate-to-extreme discomfort could be contributed to "length of compression time" (27\%) and "compression force" (53\%). As the standard breast screening involves a minimum of four breast views, these factors are particularly important to consider (Figure 3).

With respect to the breast on the platform, technologists were asked to rate the level of patient discomfort for: 1) coolness of breast platform; 2) pinching at chest wall; 3) hard edges of breast platform and 4) hard edges of paddle. "Pinching at chest wall" and "hard edges of breast platform" were cited significantly more times $(P<0.05)$ than coolness and edges of paddle (Figure 4).

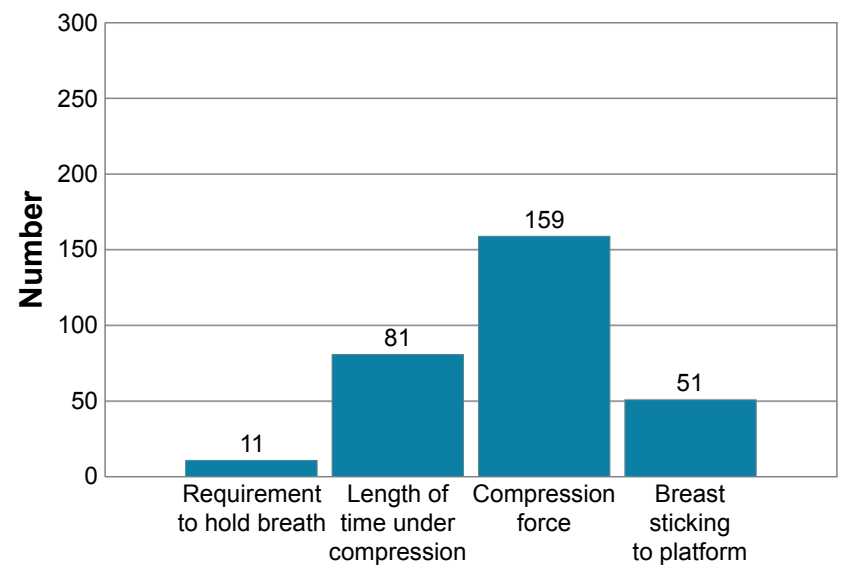

Figure 3 Number of technologists reporting patients who have "high-to-very high" discomfort based on procedural steps.

\section{Psychology of discomfort}

"Proper communication of exam expectations" was rated to have significantly more impact on patient discomfort as compared to the other options. Lower compression force and softer paddles were the features cited next as potentially having the most impact on patient discomfort (Figure 5).

These findings are consistent with past research, which reported that the level of care a patient receives from the technologist may outweigh the actual physical discomfort experienced during the examination. This finding suggests that the psychological aspect of an examination is just as important, if not more important, than physical aspects. Similarly, in a study focused on the patient experience during magnetic resonance imaging (MRI) ${ }^{15}$ patients reported that a trustful dialog with the radiographer was crucial in helping to manage "fear, discomfort and feelings of loss of self-control".

Although patients have reported experiencing intense pain or discomfort in past studies, it is noteworthy that studies

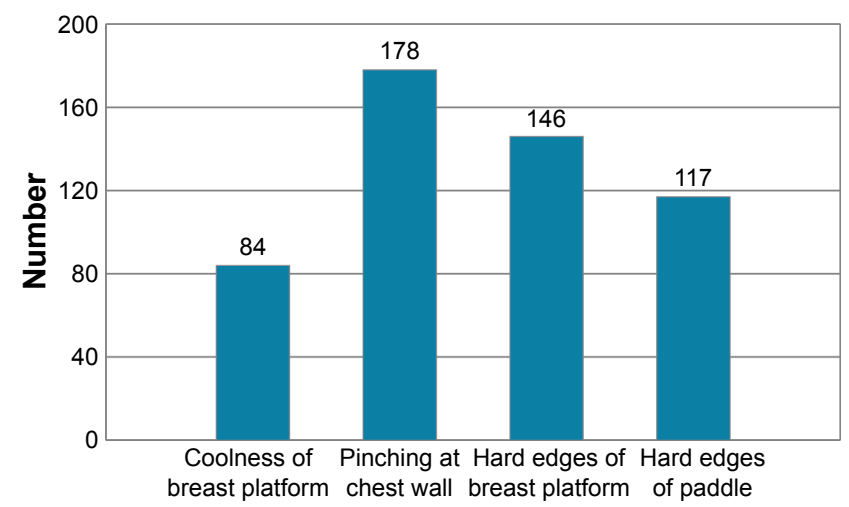

Figure 4 Number of technologists reporting patients who have "high-to-very high" discomfort from physical contact with the breast platform. 


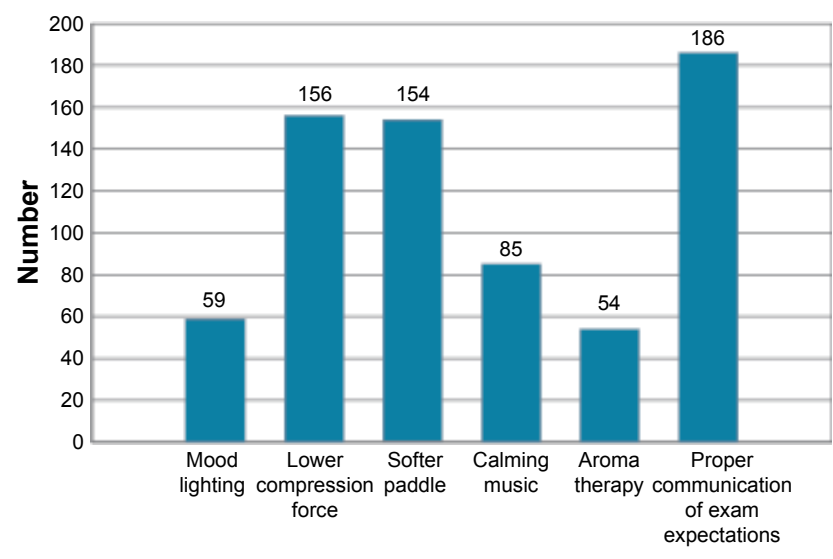

Figure 5 Number of technologists reporting "high-to-very high" on how specific factors may affect patient discomfort.

have also documented that patients report their experience to be less painful than anticipated. ${ }^{3}$ In the same study, $26 \%$ of patients reported anxiety as a factor that may interfere with scheduling their subsequent screens - an example of how psychology affects both the perception of pain and attitudes toward mammography in general.

\section{Addressing discomfort}

The results from this study used the technologists' independent assessments of patient discomfort, which appear to confirm a number of findings from previous studies ${ }^{3-5,7,9-12}$ that reported discomfort and anxiety to be prevalent in various patient populations. The findings suggest that from a patient demographic perspective, Asian women, patients with dense breasts and those who have had previous procedures experienced significantly more discomfort than other demographic groups. In addition, the act of compression, coupled with length of compression time, continues to be a primary contributor to discomfort.

In a study on positron emission tomography (PET)/MRI, patient comfort during examination was reported to be "mandatory" for patient compliance and for image quality. ${ }^{16}$ This need for improved comfort was accomplished by shorter examination times and fairly comfortable positioning. This study found that $26 \%$ of technologists reported that discomfort associated with mammography impacts patient satisfaction and that $36 \%$ of the technologists believe that "fear of pain during a mammogram" impacts screening compliance (Figure 6).

Although the imaging modality in the study of Stahr et al is different, the idea of patient comfort as a core requirement is an important consideration for mammography practices. The findings of this and previous studies on other modalities suggest that by increasing technologist-patient communication, considering examination equipment (eg, paddle and platform

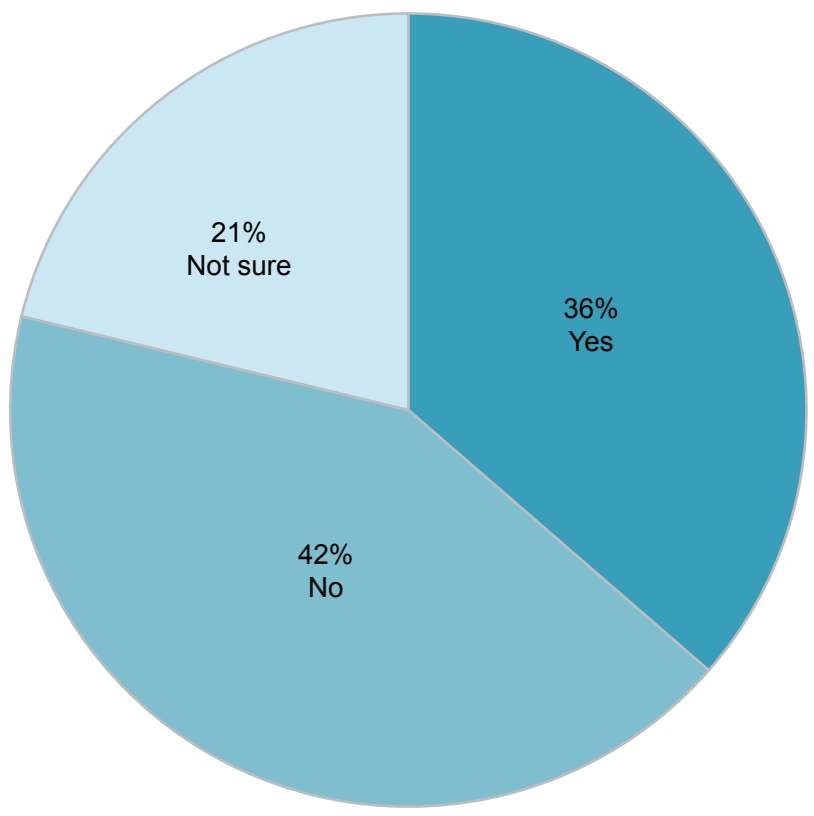

Figure 6 Proportion of technologists who report that "fear of pain during a mammogram" impacts screening compliance.

shapes) related to comfort and using more comfortable positioning techniques, patient discomfort in breast imaging centers could be decreased. Although compression is necessary to stabilize the breast, other viable approaches may affect "perceived" patient comfort.

One such approach is the use of radiolucent breast cushion products already available in the market. In a study by Miller et $\mathrm{al},{ }^{17}$ the use of breast cushions was found to decrease the amount of discomfort experienced by patients. At sites that did not use a breast cushion as the standard of care, technologists who had access to breast cushions reported using the pads primarily for patients who expressed anxiety over the pending examination. Based on the aforementioned findings, it is likely that more frequent use of breast pads would decrease the amount of discomfort and support screening compliance.

In addition to the use of breast cushions, breast imaging centers wanting to positively impact routine screening participation may want to consider other methods to increase psychological and physical comfort. One such method is to develop initiatives to maximize time in room with patients, facilitating improved communication. While increased communication with the patient may increase upfront time, it may reduce positioning time, as patients are more comfortable and understand examination expectations.

\section{Study limitations}

Although the research in this study revealed a number of findings and opportunity areas from a unique perspective 
(the technologist point of view of the patients' experiences), there were a number of study limitations that should be noted. The findings of this study were limited to perceptions that technologists have regarding the patient experience with respect to patient discomfort. This research was not validated by actual patient data.

As a survey-based study, limited information was available regarding the respondents themselves. For instance, detailed facility information was not captured, in which case it is not known whether geography or facility type could have been skewed in one direction. Survey research is based on recall, which is based on memory and past experiences. To this end, some participants may have developed perceptions or biases overtime that cannot be readily detected in surveys.

\section{Conclusion}

This study provides a technologist perspective regarding the "whys" of patient discomfort during imaging. This is a unique perspective, as little to no research has been conducted to date using this perspective. The findings from the study confirm some of what has been reported to date and challenge other findings.

The findings from this study suggest that multiple factors, including psychological and physical, ethnicity and age, may impact breast discomfort during mammography. Breast imaging system manufacturers and centers should consider approaches to reduce discomfort - both perceived and physical - in order to promote screening compliance and ultimately early detection. Breast imaging centers may benefit from focusing continuing medical education activities around positioning technique and communication. Although more research is needed, it is apparent that the patient experience of comfort and pain during mammography is an area warranting increased attention, research and solutions.

\section{Disclosure}

The authors are employees or consultants to Hologic, Inc. The authors report no other conflicts of interest in this work.

\section{References}

1. American Cancer Society. Breast Cancer Facts and Figures 2015-1016. 2015. Available from: http://www.cancer.org/acs/groups/content/@ research/documents/document/acspc-046381.pdf. Accessed October 31, 2016.

2. Centers for Disease Control and Prevention (CDC). Health, United States. Atlanta, GA: Centers for Disease Control and Prevention (CDC); 2015. Table 70.

3. Sapir R, Patlas M, Strano SD, Hadas-Halpern I, Cherny NI. Does mammography hurt? J Pain Symptom Manage. 2003;25(1):53-63.

4. Padoan M, Ferrante D, Pretti G, Magnani C. Study of socio-economic characteristics, diagnosis and outcome of women participating or not participating in mammogram screening. Ann Ig. 2014;26(6):518-526.

5. De Groot JE, Branderhorst W, Grimbergen CA, den Heeten GJ, Broeders MJM. Towards personalized compression in mammography: a comparison study between pressure- and force-standardization. Eur J Radiol. 2015;84(3):384-391.

6. Rutter DR, Calnan M, Vaile MS, Field S, Wade KA. Discomfort and pain during mammography: description, prediction, and prevention. BMJ. 1992;305(6851):443-445.

7. Kornguth PJ, Keefe FJ, Conaway MR. Pain during mammography: characteristics and relationship to demographic and medical variables. Pain. 1996;66(2):187-194.

8. Bruyninckx E, Mortelmans D, Van Goethem M, Van Hove E. Risk factors of pain in mammographic screening. Soc Sci Med. 1999;49(7):933-941.

9. Keemers-Gels ME, Groenendijk RPR, Heuvel JHM, et al. Pain experienced by women attending breast cancer screening. Breast Cancer Res Treat. 2000;60(3):235-240.

10. Engelman KK, Cizik AM, Ellerbeck EF. Women's satisfaction with their mammography experience: results of a qualitative study. Women Health. 2005;42(4):17-35.

11. Whelehan P, Evans A, Wells M, MacGillivray S. The effect of mammography pain on repeat participation in breast cancer screening: a systematic review. Breast. 2013;22(4):389-394.

12. Asghari A, Nicholas MK. Pain during mammography: the role of coping strategies. Pain. 2004;108(1):170-179.

13. Sickles EA, D’Orsi CJ, Bassett LW, et al. ACR BI-RADS® mammography. ACR BI-RADS® Atlas, Breast Imaging Reporting and Data System. Reston, VA: American College of Radiology; 2013.

14. Straker LM. Body discomfort assessment tools. In: Karwowski W, Marras W, editors. The Occupational Ergonomics Handbook. New York: CRC Press, LLC; 1999:26-1-26-13.

15. Carlsson S, Carlsson E. 'The situation and the uncertainty about the coming result scared me, but interaction with the radiographers helped me through': a qualitative study on patients' experiences of magnetic resonance imaging examinations. J Clin Nurs. 2013;22(21-22):3225-3234.

16. Stahr K, Poulson JM, Federspiel M, et al. Patient comfort and compliance in combined PET/MR imaging. J Nucl Med. 2013;54(2):2530.

17. Miller D, Livingstone V, Herbison GP [webpage on the Internet]. Interventions for relieving the pain and discomfort of screening mammography. In: The Cochrane Collaboration, editor. Cochrane Database of Systematic Reviews. Chichester, UK: John Wiley \& Sons, Ltd; 2008. Available from http://doi.wiley.com/10.1002/14651858.CD002942. pub2. Accessed October 31, 2016.
International Journal of Women's Health

\section{Publish your work in this journal}

The International Journal of Women's Health is an international, peerreviewed open-access journal publishing original research, reports, editorials, reviews and commentaries on all aspects of women's healthcare including gynecology, obstetrics, and breast cancer. The manuscript management system is completely online and includes

\section{Dovepress}

a very quick and fair peer-review system, which is all easy to use. Visit http://www.dovepress.com/testimonials.php to read real quotes from published authors. 